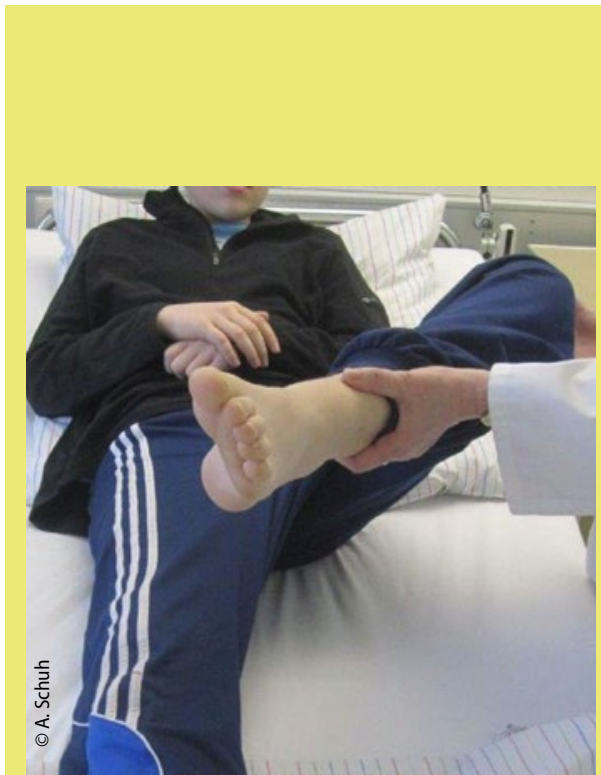

Abb. 1 Drehmann-Zeichen: Passive Beugung des Knie- und Hüftgelenks erzwingt Außenrotation im Hüftgelenk.
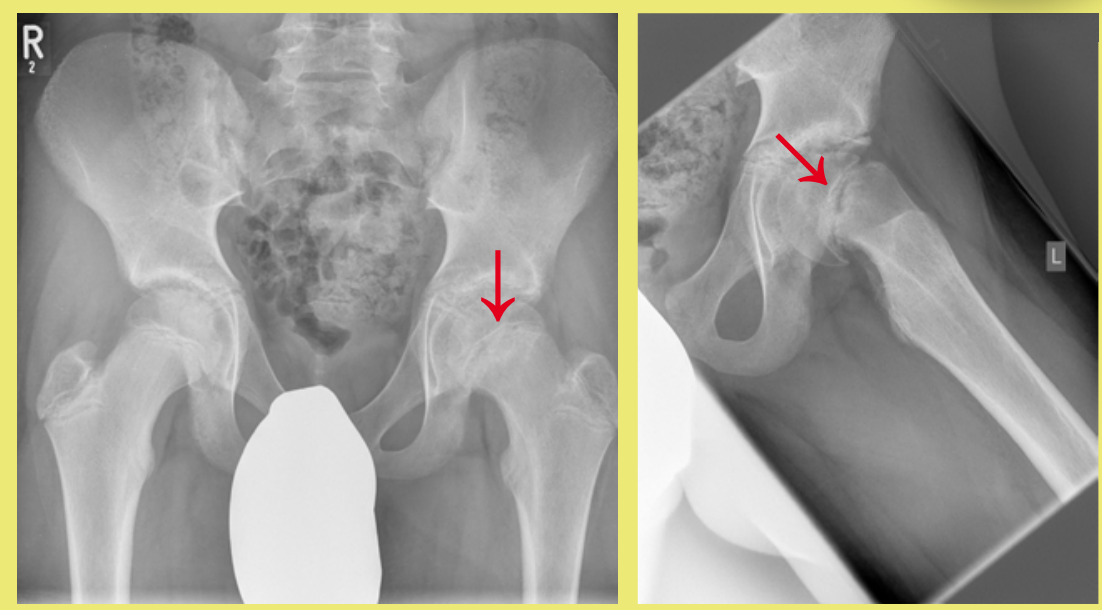

Abb. 2a, 2b Röntgenaufnahmen des Beckens und der linken Hüfte in Lauensteinposition mit Epiphysenlösung des Femurkopfs links (Pfeile).

\title{
Kinderothopädischer Notfall
}

\section{Beim Beugen macht das Bein nicht mit}

Ein 15 Jahre alter Patient stellte sich mit seit fünf Wochen bestehenden Hüftschmerzen links vor. Die körperliche Untersuchung zeigte ein schonhinkendes Gangbild links. Die Beweglichkeit der linken Hüfte war mit Extension/Flexion 0/0/90 Abduktion/Adduktion $20 / 0 / 20^{\circ}$ und Außenrotation/Innenrotation $30 / 10 / 0^{\circ}$ eingeschränkt. Wir prüften nun das sogenannte Drehmann-Zeichen. Der Patient sollte sich auf den Rücken legen, damit ein Arzt das Bein am Fuß und in Höhe des Kniegelenks fassen und passiv im Hüftgelenk beugen konnte. Dies gelang nur bei gleichzeitiger Abduktion und Außenrotation (Abb. 1) - das Zeichen war somit positiv.

Röntgenaufnahmen des Beckens (Abb. 2a) sowie der linken Hüfte in Lauensteinposition (Abb. 2b) bestätigten die Verdachtsdiagnose einer Epiphyseolysis capitis femoris (ECF), also eines Abrutschens des Hüftkopfs nach hinten unten. Die zweite Ebene ist in diesem Fall besonders wichtig, da die Krankheit häufig nur so zu erkennen ist. Noch am selben Tag wurde die linke Hüfte reponiert und mittels Kirschnerdraht fixiert. Auch die rechte Hüfte wurde prophylaktisch fixiert.

Gerade bei Jugendlichen ist das Drehmann-Zeichen bei Hüftgelenkserkrankungen positiv. Meist deutet es auf eine ECF hin, viel seltener auf Entzündungen, einen fortgeschrittenen Morbus Perthes oder eine deformierende Coxarthrose. Die
ECF tritt typischerweise erstmals zwischen dem 10. und dem 14. Lebensjahr auf. Jungen sind dreimal häufiger betroffen als Mädchen. Viele der Patienten sind übergewichtig,

Die Epiphysenlösung tritt entweder schleichend oder plötzlich als schmerzhaftes Ereignis ein. Möglich ist auch ein akutes Ereignis bei vorbestehender allmählicher Verschiebung. Die akute ECF ist einer der wenigen Notfälle in der Kinderorthopädie - und wird trotzdem häufig übersehen.

Um eine spätere Fehlform der Hüfte (Coxa vara retrotorta) zu verhindert, sollte schnellstmöglich operiert werden. Bei jungen Patienten mit noch deutlichem Restwachstum wird mit Bohr- oder Kirschnerdrähten oder auch mit kanülierten Schrauben stabilisiert. Möglich sind auch sogenannte dynamische epiphysäre Teleskopschrauben. In Deutschland wird üblicherweise auch die Gegenseite prophylaktisch stabilisiert, da mit einer Wahrscheinlichkeit von ca. 10-60\% auch die andere Hüfte später betroffen sein kann.

\section{$\rightarrow$ Prof. Dr. med. Alexander Schuh}

Muskuloskelettales Zentrum der Kliniken des Landkreises Neumarkt i. d. Oberpfalz, Nürnberger Str. 12, D-92318 Neumarkt

Dr. med. Kerstin Remmel

Abteilung für Kinderorthopädie, Cnopf'sche Kinderklinik, Nürnberg 